\title{
O uso da instrumentação tecnológica integrada para identificação de oportunidades de Tecnologias Assistivas: o caso de uma usuária cadeirante.
}

\author{
The use of integrated technological instrumentation to identify Assistive \\ Technology opportunities: the case of a wheelchair user. \\ MERINO, Giselle Schmidt Alves Díaz \\ Universidade do Estado de Santa Catarina; Universidade Federal de Santa Catarina, Dra. \\ gisellemerino@gmail.com
}

PAULO, Irandir Izaquiel

Universidade Federal de Santa Catarina, Mestrando em Design irandirpitangadesigner@gmail.com

FORCELINI, Franciele

Universidade Federal de Santa Catarina, Doutoranda em Design

francieleforcelini@gmail.com

\author{
VARNIER, Thiago \\ Universidade Federal de Santa Catarina, Doutorando em Design \\ thiagovarnier1@gmail.com
}

\section{RESUMO}

Instrumentos tecnológicos vêm sendo utilizados como recursos auxiliares para o levantamento de dados precisos e quantificáveis. Este artigo visa identificar oportunidades para projetos de tecnologias assistivas para um sujeito com deficiência motora a partir da análise de dados quantitativos oriundos da termografia infravermelha e captura de movimentos (Xsens). Caracteriza-se como um estudo aplicado, qualitativo, com objetivo exploratório, descritivo e correlacional. Utiliza-se da instrumentação tecnológica para avaliar um sujeito com deficiência, com base em protocolos de coletas que possibilitam manter o rigor científico e a confiabilidade dos dados. Os resultados apontam oportunidades de desenvolvimento de dispositivos assistivos para redução da pressão coxofemoral, proteção para os joelhos, transferência para a cadeira de rodas, bem como assentos personalizados, novos revestimentos e um sistemas de propulsão mais eficiente para cadeira de rodas. Contudo, evidencia-se que a instrumentação tecnológica possibilita dados precisos mesmo quando as coletas envolvem sujeitos com limitações, auxiliando na compreensão das suas necessidades reais e no desenvolvimento de projetos mais eficientes, centrados nos usuários.

Termografia infravermelha; Captura de Movimentos; Design; Tecnologia Assistiva, Deficiência Motora. 


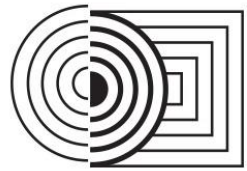

\section{$17^{\circ}$ ERGODESIGN \& USIHC 2019 \\ PUC-Rio, 11 a 13 de dezembro \\ Rio de Janeiro, RJ, Brasil}

$17^{\circ}$ Ergodesign - Congresso Internacional de Ergonomia e Usabilidade de Interfaces Humano Tecnológica: Produto, Informações Ambientes Construídos e Transporte

$17^{\circ}$ USIHC - Congresso Internacional de Ergonomia e Usabilidade de Interfaces Humano Computador

\begin{abstract}
Technological instruments have been used as auxiliary resources for gathering accurate and quantifiable data. This paper aims to identify opportunities for assistive technology projects for a motor-impaired subject from the analysis of quantitative data from infrared thermography and motion capture (Xsens). This study is characterized as applied, qualitative and with exploratory, descriptive and correlational objective. Technological instrumentation is used to evaluate a subject with disabilities, based on data collection protocols that allow maintaining scientific rigor and data reliability. The results highlight opportunities for developing assistive devices for lower hip pressure, knee protection, wheelchair transfer, as well as custom seating, new coverings and a more efficient wheelchair propulsion system. However, it is evident that technological instrumentation enables accurate data even when collections involve subjects with limitations, helping to understand their real needs and the development of more efficient, user-centered designs.
\end{abstract}

Infrared Thermography; Motion Capture; Design; Assistive Technology; Motor Disability.

\title{
1. INTRODUÇÃO
}

Tecnologia refere-se ao conhecimento e a aplicação de ferramentas, técnicas e ciências, com o propósito de dar forma ao mundo (BEST, 2012). Engloba o conhecimento, estudo e domínio sobre um determinado modo específico de operar ou fazer algo (CIPINIUK; PORTINARI, 2011). As tecnologias e as inovações impactam nas estruturas de todos os níveis da Gestão de Design, modificando a forma como interagem, viabilizam e comportam novas relações, processos e práticas, proporcionando oportunidades para o design (BEST, 2012).

Deste modo, o processo de design pode se apropriar de recursos como os instrumentos tecnológicos ${ }^{1}$ para a coleta de dados objetivos e quantificáveis, que podem aproximar a equipe de projeto das necessidades reais dos usuários, bem como auxiliar no desenvolvimento de novos produtos (MERINO et al., 2017b; MERINO et al, 2018). Esses instrumentos podem oferecer agilidade ao processo, gerando dados confiáveis que podem tornar os projetos mais eficientes e satisfatórios (FORCELINI; VARNIER; MERINO, 2018). Além disso, os dados quantificáveis e precisos sobre o problema ou necessidade atendida podem minimizar as incertezas do projeto (MERINO et al., 2017; SPECK et al., 2016).

Em coletas com pessoas com deficiência, os instrumentos tecnológicos assumem um papel ainda mais significativo, auxiliando na obtenção de dados dos sujeitos com diferentes incapacidades (MERINO et al., 2017; SPECK et al.,2016). Possibilitam a obtenção de dados precisos mesmo quando a comunicação com os sujeitos se encontra comprometida (por limitações físicas e psíquicas), impedindo o levantamento de informações por meios como entrevistas, questionários, entre outros (MERINO et al., 2017). A instrumentação permite uma exploração aprofundada dos sujeitos e objetos em estudo (FORCELINI; VARNIER; MERINO, 2019), bem como a aproximação entre a equipe de projeto e as capacidades e limitações do usuário, fazendo com que os projetos promovam melhorias na qualidade de vida de pessoas com deficiência ou mobilidade reduzida (MERINO et al., 2018; VARNIER; MERINO, 2018).

Neste sentido, Merino et al. (2018) destacam que a captura de movimentos (Xsens) e a termografia infravermelha (TIV) são métodos viáveis para o desenvolvimento de tecnologias assistivas (TAs), promovendo soluções mais eficientes, confortáveis e seguras. Essas

\footnotetext{
${ }^{1}$ Entende-se por instrumentos tecnológicos equipamentos dotados de distintas tecnologias, os quais são capazes de obter medições precisas e dados quantificáveis.
} 


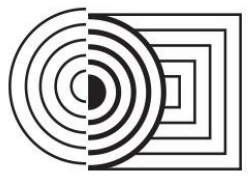

\section{$17^{\circ}$ ERGODESIGN \& USIHC 2019 \\ PUC-Rio, 11 a 13 de dezembro \\ Rio de Janeiro, RJ, Brasil}

$17^{\circ}$ Ergodesign - Congresso Internacional de Ergonomia e Usabilidade de Interfaces Humano Tecnológica: Produto, Informações Ambientes Construídos e Transporte

$17^{\circ}$ USIHC - Congresso Internacional de Ergonomia e Usabilidade

de Interfaces Humano Computador

tecnologias podem auxiliar no desenvolvimento de novos projetos, nas adaptações de produtos existentes, na identificação de problemas e em análises ergonômicas (FORCELINI; VARNIER; MERINO, 2019). Sendo assim, mediante as potencialidades dessas tecnologias, este artigo tem como objetivo identificar oportunidades para projetos de tecnologias assistivas para um sujeito com deficiência motora a partir da análise de dados quantitativos oriundo da termografia infravermelha e da captura de movimentos.

\section{FUNDAMENTAÇÃO TEÓRICA}

\subsection{Termografia Infravermelha}

A termografia infravermelha é um método de detecção da radiação infravermelha emitida pelas superfícies, o qual possibilita o registro das qualidades térmicas de objetos e sujeitos por meio das imagens térmicas geradas (termogramas), que exprimem as variações de temperatura (imperceptíveis a olho nu) por um espectro cromático (CERDEIRA et al, 2011; GABRIEL et al., 2016; MOBLEY, 2002; SILVA, 2017).

Caracteriza-se como um método não destrutivo, sem contato direto, e seus dados podem ser comparados e analisados por meio de softwares específicos, normalmente associados aos fornecedores das câmeras termográficas (GABRIEL et al., 2016; HOLST, 2000; MOBLEY, 2002; SILVA, 2017). Destaca-se pela versatilidade, podendo ser utilizada para analisar temperaturas cutâneas (BRIOSCHI; MACEDO, 2003), auxiliar no desenvolvimento e avaliação de produtos (FORCELINI; MERINO, 2019), detectar a deterioração de produtos e defeitos em materiais (SILVA; TARALLI; MELZ, 2015; SILVA; MIRA, 2016), bem como para indicar condições atípicas em qualquer sistema por meio da variação da sua temperatura superficial (CERDEIRA et al., 2011).

No que se refere a aplicação da TIV, Forcelini e Merino (2019) destacam as tecnologias assistivas como a categoria de produtos mais analisada por meio desta tecnologia, demonstrando sua efetividade em projetos envolvendo sujeitos com limitações físicas e psíquicas, devido a sua capacidade de detectar e quantificar temperaturas (dados precisos) de maneira não invasiva e sem interferências. A TIV pode permitir a compreensão do estado muscular do sujeito, podendo auxiliar no desenvolvimento e avaliação de produtos por meio de uma exploração aprofundada e menos subjetiva (MERINO et al., 2018).

\subsection{Captura de movimentos}

A Captura de Movimentos, também chamada de Motion Tracking, trata-se de um método utilizado para mapear os movimentos realizados por um objeto e reproduzi-los em ambiente digital (GOMIDE et al., 2009). Tem a finalidade de registrar o movimento de um corpo por meio de algum dispositivo e, a partir dos dados registrados, calcular as variáveis cinemáticas do movimento (STREIT, 2013).

Dentre os sistemas de captura existentes, o Xsens é um sistema baseado em unidades de medidas inerciais, que combina sinais tridimensionais provenientes de giroscópio e acelerômetros para rastrear o posicionamento do objeto capturado, determinando sua velocidade, aceleração e posição (XSENS, 2012). Neste sistema, os giroscópios são utilizados para medir a orientação dos segmentos corporais e a velocidade de rotação de um objeto em torno de um eixo central. Ou seja, consegue mensurar a velocidade angular, que permite definir o ângulo e orientação dos objetos (CARVALHO, 2011). Os acelerômetros, por sua vez, são 


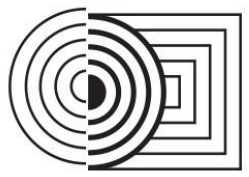

\section{$17^{\circ}$ ERGODESIGN \& USIHC 2019 \\ PUC-Rio, 11 a 13 de dezembro \\ Rio de Janeiro, RJ, Brasil}

$17^{\circ}$ Ergodesign - Congresso Internacional de Ergonomia e Usabilidade de Interfaces Humano Tecnológica: Produto, Informações Ambientes Construídos e Transporte

$17^{\circ}$ USIHC - Congresso Internacional de Ergonomia e Usabilidade

de Interfaces Humano Computador

dispositivos eletromecânicos que medem a aceleração e a velocidade do objeto, calculando as forças exercidas sobre ele (YANG; HSU, 2010).

O sistema Xsens capta movimentos em tempo real por meio de 17 sensores inerciais e magnéticos com frequência de $120 \mathrm{~Hz}$ (120 frames por segundo), o que possibilita a avaliação contínua do movimento dinâmico (XSENS, 2012). Logo, os dados obtidos são transmitidos por conexão wireless para um computador com um software que permite a observação, gravação e análise dos movimentos a partir de gráficos dos ângulos das articulações, da velocidade e duração dos movimentos (SPECK et al., 2016; ROETENBER; LUINGE; SLYCKE, 2013).

Varnier e Merino (2018) apontam que a captura de movimentos facilita o levantamento de informações precisas e quantificáveis dos movimentos de segmentos e articulações, tornandose um complemento positivo para as medidas subjetivas. Desta forma, sua utilização possibilita a criação de novos produtos e auxilia na identificação dos requisitos de projeto, podendo ser uma ferramenta eficiente para a obtenção de dados quantitativos na área de design, gerando informações e definições precisas da biomecânica (VARNIER; MERINO, 2018).

\subsection{Tecnologia Assistiva}

No mundo, um em cada sete indivíduos possuem algum tipo de deficiência (motora, sensorial e/ou cognitiva) e, no Brasil, isso corresponde a 45 milhões de indivíduos (BRASIL, 2012; WHO, 2012). Sendo assim, o uso e o desenvolvimento de TAs vem se tornando cada vez mais necessário. São produtos, recursos, estratégias, práticas, processos, métodos e serviços com a finalidade de promover a funcionalidade de indivíduos com deficiência ou mobilidade reduzida, visando sua autonomia, independência, qualidade de vida e inclusão social (BRASIL, 2015). As TAs caracterizam-se como um subsídio na melhoria das capacidades funcionais negativas dos indivíduos, que possibilita a realização da atividade desejada, antes bloqueada por algum tipo de deficiência ou envelhecimento (BERSCH, 2017).

No que se refere ao desenvolvimento de projetos de TAs, a atuação do designer proporciona produtos e/ou serviços favoráveis ao ambiente no qual foram inseridos, facilitado a inclusão das pessoas com deficiência (BASSO, 2012). Para o desenvolvimento de projetos, o usuário deve ser totalmente incluído, considerando-se seu contexto de vida, suas capacidades funcionais e habilidades (BERSCH, 2017). Esse conhecimento sobre o potencial motor, sensorial e cognitivo do usuário possibilita a avaliação TAs existentes e o desenvolvimento de novos projetos para uma necessidade específica (BERSCH, 2017). Assim, os dispositivos assistivos podem suprir uma potencialidade comprometida, diminuir as implicações de uma alteração gradativa, reduzir a carência de cuidadores, bem como evitar ocorrências de outras enfermidades (WHO, 2012).

\section{PROCEDIMENTO METODOLÓGICOS}

O presente estudo possui natureza aplicada e interesse prático, uma vez que visa utilizar seus resultados para a solução de problemas reais (MARCONI; LAKATOS, 2007). Nesse sentido, visa identificar oportunidades de projeto de TAs para um sujeito cadeirante com paraplegia e atrofias (membros superiores e inferiores). Possui abordagem qualitativa, por se tratar de uma tentativa de compreender detalhadamente os significados e características situacionais (MARCONI; LAKATOS, 2007) do sujeito da pesquisa. 


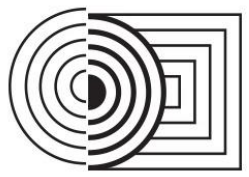

\section{$17^{\circ}$ ERGODESIGN \& USIHC 2019 \\ PUC-Rio, 11 a 13 de dezembro \\ Rio de Janeiro, RJ, Brasil}

$17^{\circ}$ Ergodesign - Congresso Internacional de Ergonomia e Usabilidade de Interfaces Humano Tecnológica: Produto, Informações Ambientes Construídos e Transporte

$17^{\circ}$ USIHC - Congresso Internacional de Ergonomia e Usabilidade de Interfaces Humano Computador

Quanto aos objetivos, classifica-se como exploratório, descritivo e correlacional. Os estudos exploratórios visam examinar um problema pouco estudado, ajudando o pesquisador a se familiarizar com fenômenos desconhecidos, obter informação de um contexto específico, pesquisar novos problemas, identificar variáveis promissoras, estabelecer prioridades para estudos futuros ou sugerir afirmações (SAMPIERI; COLLADO; LUCIO, 2013). O objetivo descritivo busca especificar as propriedades e as características de um fenômeno que se submeta a uma análise, coletando informações sobre suas variáveis, indicando como estas se relacionam (SAMPIERI; COLLADO; LUCIO, 2013). O objetivo correlacional, por sua vez, visa conhecer a relação ou grau de associação que existe entre duas ou mais variáveis em um contexto específico (SAMPIERI; COLLADO; LUCIO, 2013). Ou seja, neste estudo exploram-se as condições específicas de um sujeito com deficiência física, visando a descrição das suas condições (com base em dados gerados pelo uso da instrumentação tecnológica) e a correlação dos dados para identificar oportunidades de projeto.

Sendo assim, este estudo segmenta-se em três etapas: 1 - Levantar; 2 - Descrever e analisar e; 3 - Identificar. A primeira etapa (Levantar) contemplou a realização do levantamento por meio da instrumentação tecnológica - termografia infravermelha e captura de movimentos -, registros fotográficos, de vídeo e observações assistemáticas. Na segunda etapa (Descrever e Analisar) foi realizada a análise dos dados levantados na etapa anterior com o intuito de realizar a terceira etapa (Identificar). Essa última etapa visou correlacionar os dados e identificar oportunidades para o desenvolvimento de projetos de TAs para o sujeito analisado.

\subsection{Materiais e métodos}

A coleta de dados (etapa 1 - Levantar) foi realizada com um sujeito com deficiência (paraplegia, encurtamento dos tendões e sensibilidade do lado esquerdo do corpo), do sexo feminino, com 24 anos de idade, usuário de cadeira de rodas. No que se refere ao sujeito da pesquisa, cabe salientar que este foi informado sobre o objetivo do estudo, consentindo com a sua realização por meio da assinatura do Termo de Consentimento Livre e Esclarecido (TCLE). Ainda, por se tratar de um estudo envolvendo um ser humano, a coleta encontra-se associada a um projeto aprovado pelo Comitê de Ética em Pesquisa com Seres Humanos (CEPSH) da Universidade Federal de Santa Catarina $\left(n^{\circ} 2.732 .152\right)$. As coletas foram realizadas na Universidade Federal da Paraíba, no campus de Rio Tinto e, para tanto, foram utilizados procedimentos específicos para cada método - termografia infravermelha e captura de movimentos.

Para a realização da coleta com a termografia infravermelha foi utilizada uma versão preliminar do protocolo Thermos (FORCELINI, 2019; FORCELINI; MERINO, 2019), que tem o objetivo de guiar o processo da coleta termográfica, manter seu rigor e confiabilidade dos dados obtidos. Desta forma, foram registrados dados sobre o contexto de coleta (layout do ambiente, variáveis ambientais e equipamentos), o usuário (sujeito da pesquisa) e o produto (cadeira de rodas).

No que se refere ao contexto da coleta, o espaço foi organizado conforme os layouts apresentados na Figura 1, considerando o posicionamento do fundo preto em TNT (01), do tripé com a câmera termográfica (02), da usuária/sujeito (03) e do produto - cadeira de rodas (04).

Figura 1 - Layout da coleta de dados termográficos. 


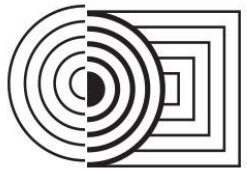

$17^{\circ}$ ERGODESIGN \& USIHC 2019

PUC-Rio, 11 a 13 de dezembro

Rio de Janeiro, RJ, Brasil $17^{\circ}$ Ergodesign - Congresso Internacional de Ergonomia e Usabilidade de Interfaces Humano Tecnológica: Produto, Informações Ambientes Construídos e Transporte

$17^{\circ}$ USIHC - Congresso Internacional de Ergonomia e Usabilidade de Interfaces Humano Computador
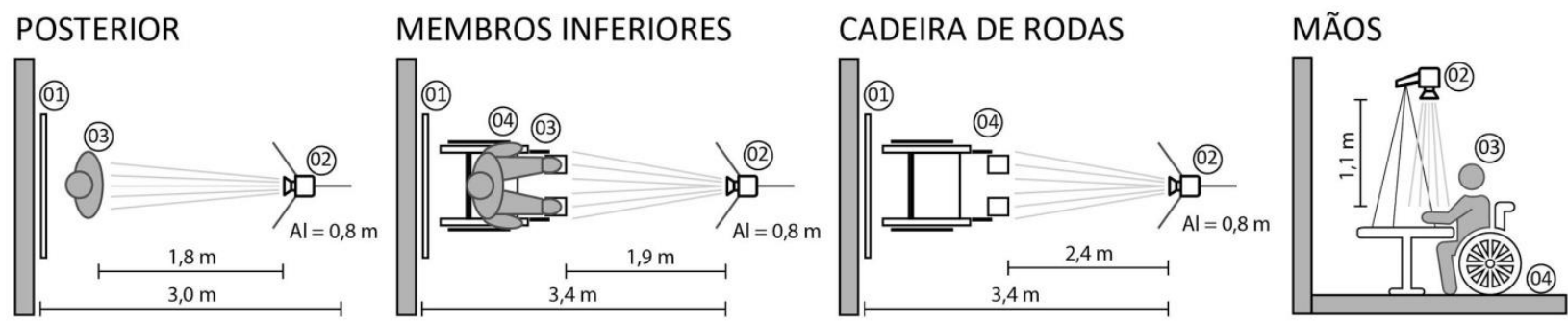

Fonte: os autores.

As variáveis ambientais foram controladas durante toda a coleta, mantendo-se a temperatura em $25^{\circ} \mathrm{C}$, a umidade do ar em $55 \%$ e a velocidade do ar em $0 \mathrm{~m} / \mathrm{s}$. Para as medições ambientais foi utilizado o Termo-Higro-Anemômetro THAL 300, da Instrutherm e, para o registro dos dados termográficos, o termovisor Flir E40 (com calibração automática).

Quanto o sujeito, foram definidas três áreas para análise - mãos (1), costas (2) e membros inferiores (3) -, as quais passaram por um período de aclimatização de 15 minutos, conforme sugere Alves Neto et al. (2009). Após a aclimatização, foi realizado o primeiro registro de cada região, em repouso (TR), e o segundo imediatamente após a realização da atividade proposta (T0). Para o registro das mão e costas, o sujeito foi orientado a deslocar-se utilizando a cadeira de rodas por um trajeto de 10 metros, sem desníveis (duração aproximada de 4 minutos). Já para o registro da região dos membros inferiores (T0), o sujeito foi orientado a deslocar-se por cerca de 5 metros sem o uso da cadeira de rodas (imagem 2), do modo com que costuma deslocar-se diariamente. Essa distância limitada foi determinada em função da sua dificuldade de deslocamento. Além destes, também foi realizado o registro da cadeira de rodas utilizada pelo sujeito (posição frontal).

Após a coleta, os dados termográficos foram extraídos por meio do software Flir Tools e organizados em planilhas do Microsoft Excel para a identificação das temperaturas dos pontos hiper radiantes, as médias e as diferenças das temperaturas nos diferentes tempos. Os parâmetros adotados para a padronização das imagens termográficas são apresentados na Tabela 1.

Tabela 1 - Parâmetros para padronização das imagens termográficas.

\begin{tabular}{|c|c|c|c|c|c|}
\hline Emissividade & $0,98^{*}$ & \multicolumn{4}{|c|}{ *Padrão para medições de temperatura da pele (GABRIEL et al., 2016). } \\
\hline Temp. Refl. & $20^{\circ} \mathrm{C}$ & Temp. atmosférica & $25^{\circ} \mathrm{C}$ & Trans. da óptica ext. & 1 \\
\hline Distâncias & Variadas (Figura 1) & Temp. da óptica ext. & $\stackrel{\circ}{ } \mathrm{C}$ & Umidade relativa & $55 \%$ \\
\hline
\end{tabular}

Fonte: os autores.

Para a captura de movimentos, foi utilizado uma versão prévia do protocolo Motion Capture (VARNIER, 2019; VARNIER; MERINO, 2019), que visa guiar o uso do equipamento MVN Biomech, da Xsens. Este sistema é baseado em unidades de medida inercial, utilizando 17 sensores que permitem registar o movimento 3D de 23 segmentos corporais e 22 articulações, bem como o centro de massa, em tempo real (GOMIDE et al.,2009; ROETENBERG; LUINGE; SLYCKE, 2013; XSENS, 2012). 
A captura dos movimentos foi realizada com isolamento dos membros inferiores, utilizando apenas os sensores inerciais nos membros superiores (cabeça, tronco, pélvis e braços). Dessa forma, realizou-se: (1) a mensuração das medidas do corpo; (2) a fixação dos sensores inerciais ao corpo e a ligação dos fios de captura; (3) a calibração do equipamento, seguindo a pose "Npose" - posição neutra; (4) a gravação dos movimentos e, por fim; (5) a análise dos dados cinemáticos no software.

As atividades gravadas foram: (1) transferência para cadeira de rodas; (2) transferência da cadeira de rodas para o chão; (3) girar no chão para ficar de joelhos (posição de marcha do sujeito) e; (4) caminhar de joelhos (marcha padrão do sujeito). Com relação aos tempos de gravação da captura de movimento variaram de 30 segundos a 1 minuto. Os dados coletados foram extraídos para uma planilha do Microsoft Excel, permitindo a realização da análise cinemática quanto a posição (ângulos).

Além das coletas com os instrumentos tecnológicos, também foram feitos registros fotográficos, de vídeo e observações assistemáticas. As observações assistemáticas qualitativas aconteceram por meio de um papel ativo e uma reflexão constante sobre as situações, com atenção aos detalhes, acontecimentos e interações do sujeito (SAMPIERI; COLLADO; LUCIO, 2013). Todas as análises foram realizadas por uma equipe multidisciplinar, que incluiu designers e fisioterapeutas.

\section{RESULTADOS E DISCUSSÕES}

Mediante as observações realizadas, foi possível o entendimento da rotina do sujeito da pesquisa, que se caracteriza como semi-dependente, realizando algumas de suas atividades de forma autônoma e outras com auxílio. Para permanecer sentada, o sujeito assume uma postura em w, conforme Figura 2-A. Para caminhar (Figura 2-B) em sua residência e áreas próximas, mantém-se ajoelhado (em contato direto com diferentes superfícies) e para locomover-se em ambientes externos, normalmente utiliza uma cadeira de rodas (Figura 3-A).

Figura 2 - Sujeito da pesquisa (marcha e uso da cadeira de rodas).

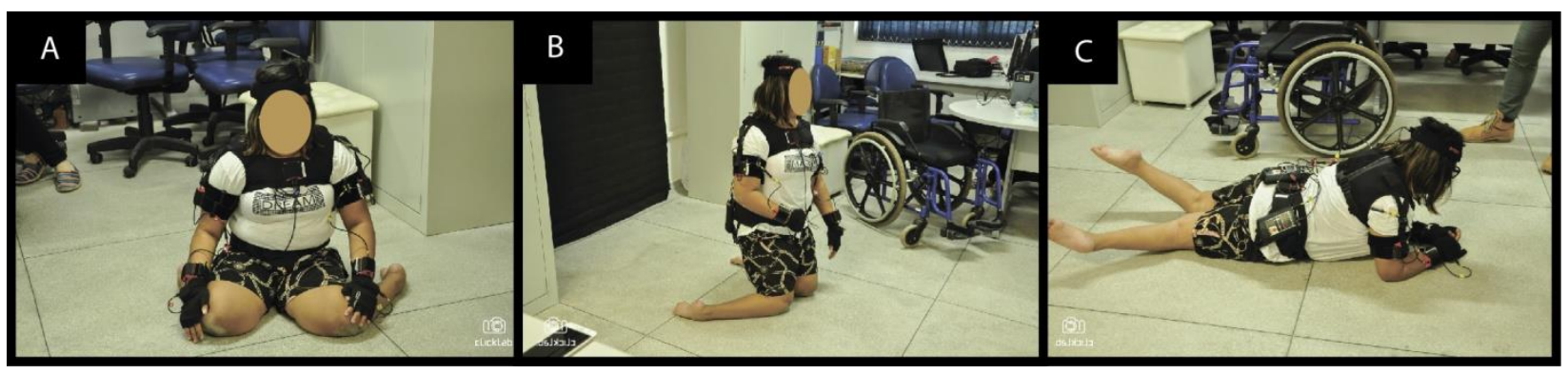

Fonte: os autores.

Ainda, possui dificuldade no processo de realização de sua transferência (chão $x$ cadeira de rodas e cadeira de rodas $x$ chão), necessitando do auxílio de uma pessoa ou mais, conforme pode ser observado na Figura 3 ( $A, B$ e $C$ ).

Figura 3 - Sujeito da pesquisa (transferência). 


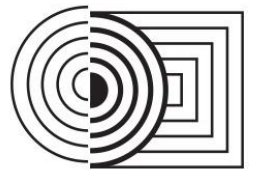

\section{$17^{\circ}$ ERGODESIGN \& USIHC 2019}

PUC-Rio, 11 a 13 de dezembro

Rio de Janeiro, RJ, Brasil

$17^{\circ}$ Ergodesign - Congresso Internacional de Ergonomia e Usabilidade de Interfaces Humano Tecnológica: Produto, Informações Ambientes Construídos e Transporte

$17^{\circ}$ USIHC - Congresso Internacional de Ergonomia e Usabilidade de Interfaces Humano Computador

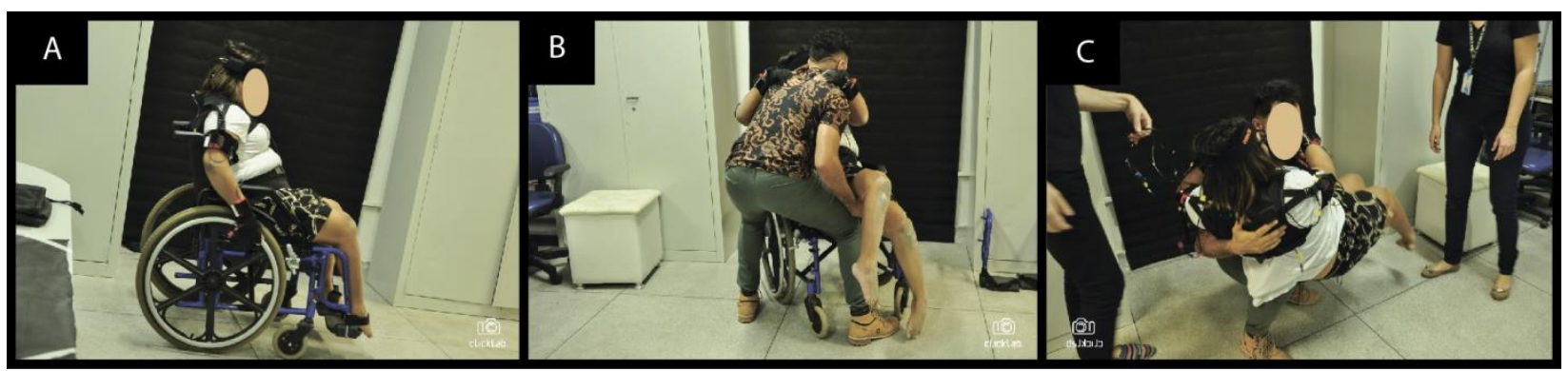

Fonte: os autores.

A transferência com auxílio requer, ao final do processo, um movimento de giro do sujeito no chão (Figura 2-C), que o coloca em uma situação de constrangimento (quando em contato com superfícies desconfortáveis e sujas) e que também gera o risco de lesões. Deste modo, destaca-se a oportunidade de desenvolvimento de um dispositivo que permita a transferência de forma autônoma e segura, eliminando a necessidade de auxílio de outras pessoas.

Ainda, após observação dos vídeos e imagens relacionadas à marcha e posição de repouso, foi verificado importante uso de rotação interna bilateral dos quadris, que pode vir a ser um fator de risco para o desenvolvimento de artrose e lesões labrais devido a acentuação do processo degenerativo articular. Este risco foi corroborado pela avaliação termográfica infravermelha da região posterior do sujeito, na qual foi verificada acentuação da temperatura na região das articulações coxofemorais (Figura 4).

Figura 4 - Termogramas da região posterior do sujeito (TR e T0).

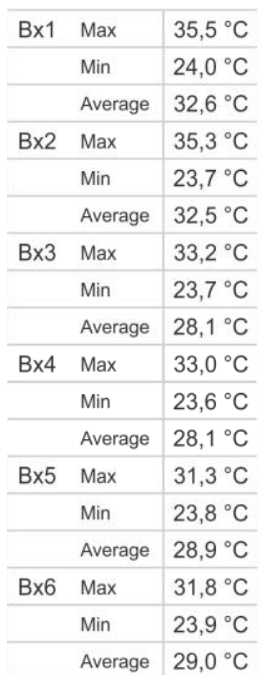

Average $29,0^{\circ} \mathrm{C}$
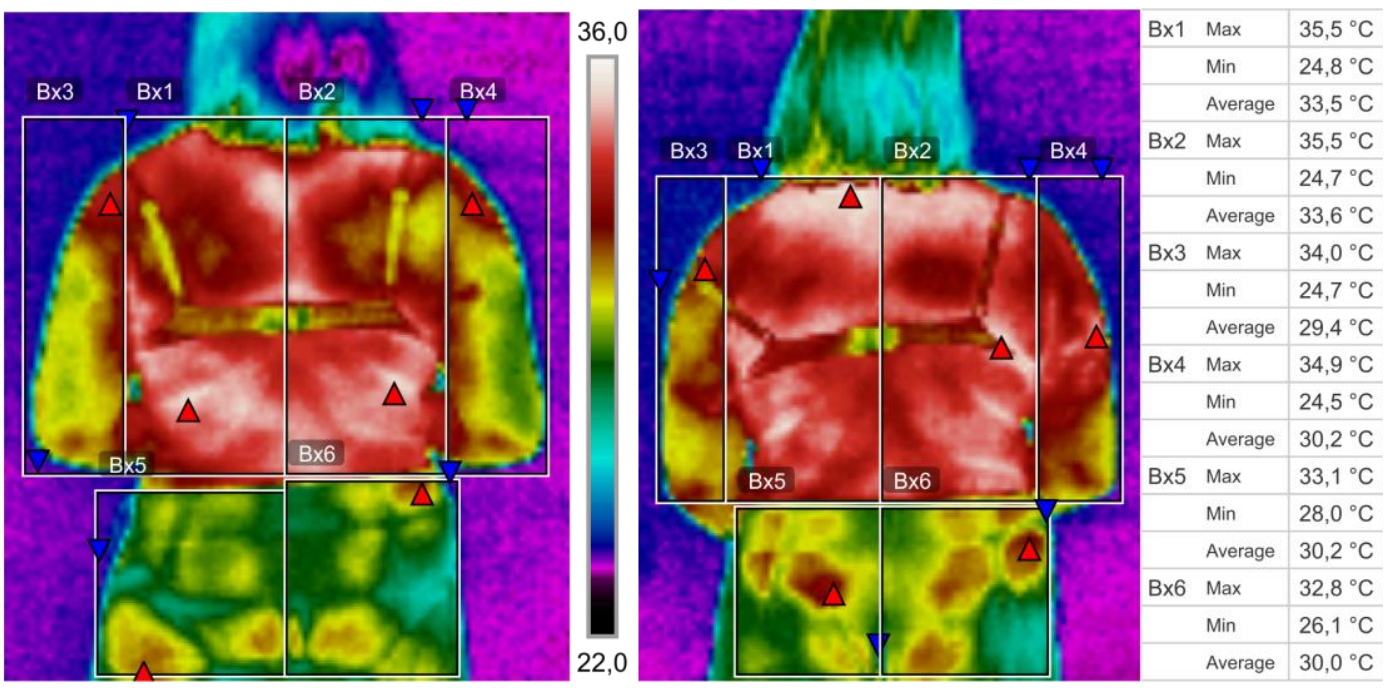

Fonte: os autores.

Após deslocamento com a cadeira de rodas, a região dos glúteos apresentou pontos hiper radiantes (maior temperatura) com temperaturas de $33,1^{\circ} \mathrm{C}$ (lado esquerdo) e $32,8^{\circ} \mathrm{C}$ (lado direito), o que representa um aumento significativo considerando que a temperatura média em repouso era $23,8^{\circ} \mathrm{C}$ (lado esquerdo) e $23,9^{\circ} \mathrm{C}$ (lado direito). Os dados termográficos demonstram o grau de vasoconstrição ou de vasodilatação da pele, que pode refletir o 
$17^{\circ}$ ERGODESIGN \& USIHC 2019

PUC-Rio, 11 a 13 de dezembro

Rio de Janeiro, RJ, Brasil $17^{\circ}$ Ergodesign - Congresso Internacional de Ergonomia e Usabilidade de Interfaces Humano Tecnológica: Produto, Informações Ambientes Construídos e Transporte

$17^{\circ}$ USIHC - Congresso Internacional de Ergonomia e Usabilidade de Interfaces Humano Computador

funcionamento, disfunção ou respostas a um traumatismo (BRIOSCHI et al., 2009). Ou seja, se houver pressão excessiva aplicada no tecido, isso pode provocar mudanças térmicas (BRIOSCHI et al., 2009).

Diante do exposto, para reduzir os riscos citados, propõe-se o desenvolvimento de um dispositivo assistivo para redução da pressão coxofemorais observada, que possibilita a estabilização e a mobilidade do sujeito de maneira confortável e segura.

Os dados termográficos da região posterior (Figura 4) também demonstraram uma elevação nas temperaturas do braço direito, deltoide direito e dos trapézios (direito e esquerdo) após a realização o deslocamento com a cadeira de rodas. $O$ braço direito apresentou elevação de $1,9^{\circ} \mathrm{C}$, onde o ponto hiper radiante passou de $33^{\circ} \mathrm{C}$ para $34,9^{\circ} \mathrm{C}$, fato que pode estar relacionado ao visível desvio da coluna do sujeito, o que pode influenciar na força aplicada de forma assimétrica para a propulsão da cadeira de rodas. Neste contexto, uma possível oportunidade de projeto seria um sistema de propulsão que reduzisse a necessidade de aplicação de força do usuário da cadeira de rodas.

Os termogramas ainda apontam o aumento das temperaturas dos trapézios bilateralmente, o que pode estar relacionado a sustentação da protrusão (anteriorização) da cabeça, como demonstram os dados da captura de movimentos das articulações T1-C7 e C1-Read (Figura 5). Este aquecimento e o do deltoide direito também podem estar associados aos movimentos de flexão e abdução dos ombros (Figura 6).

Figura 5 - Captura de movimentos do sujeito durante a marcha.

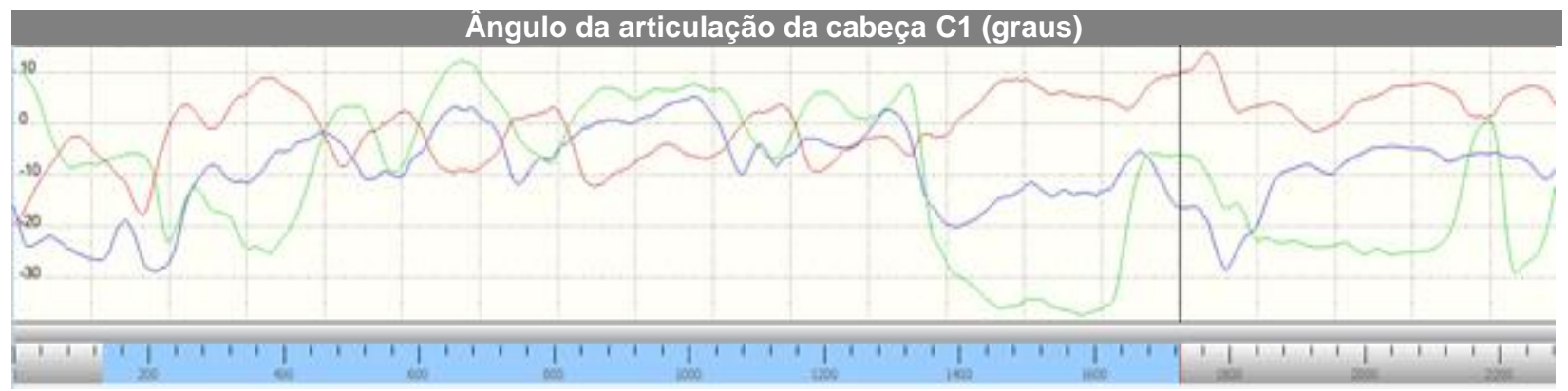

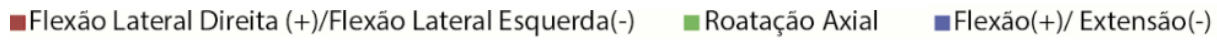

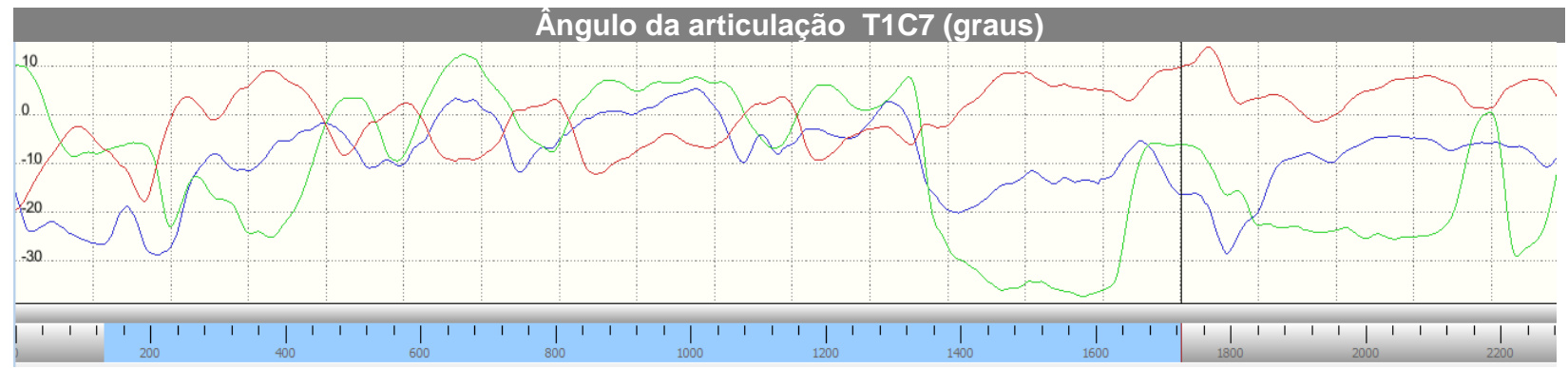

Fonte: os autores.

Em relação a análise do caminhar de joelhos (marcha padrão do sujeito) os dados obtidos com a captura de movimentos demonstram elevação na protrusão na cabeça, observado nas articulações T1-C7 e C1-Read (Figura 5), com ângulos de extensão entre 20a 30, acima da 
$17^{\circ}$ ERGODESIGN \& USIHC 2019

PUC-Rio, 11 a 13 de dezembro

Rio de Janeiro, RJ, Brasil $17^{\circ}$ Ergodesign - Congresso Internacional de Ergonomia e Usabilidade de Interfaces Humano Tecnológica: Produto, Informações Ambientes Construídos e Transporte

$17^{\circ}$ USIHC - Congresso Internacional de Ergonomia e Usabilidade

de Interfaces Humano Computador

zona de conforto estabelecida entre $0^{\circ}$ - 15ำ (TILLEY; DREYFUSS, 2005). Além disso, a manutenção da postura anteriorizada da cabeça resulta no aumento das cargas compressivas sobre a coluna cervical e pode causar a deformação dos tecidos (BONNEY; CORLETT, 2002).

Em relação a amplitude do movimento do ombro, observou-se ângulos de extensão acima de 60을 (Figura 6), o que deve ser evitado pois encontra-se fora do intervalo de conforto de $0^{\circ}-45^{\circ}$ (PALMER; EPLER, 2000; MARQUES (2003). Neste sentido, reforça-se a oportunidade para o desenvolvimento de um dispositivo que possa auxiliar na estabilização e mobilidade do sujeito, reduzindo a extensão dos ombros, bem como as lesões nos joelhos e nas articulações T1-C7, C1- Head.

Figura 6 - Captura de movimentos dos ombros (direito e esquerdo).

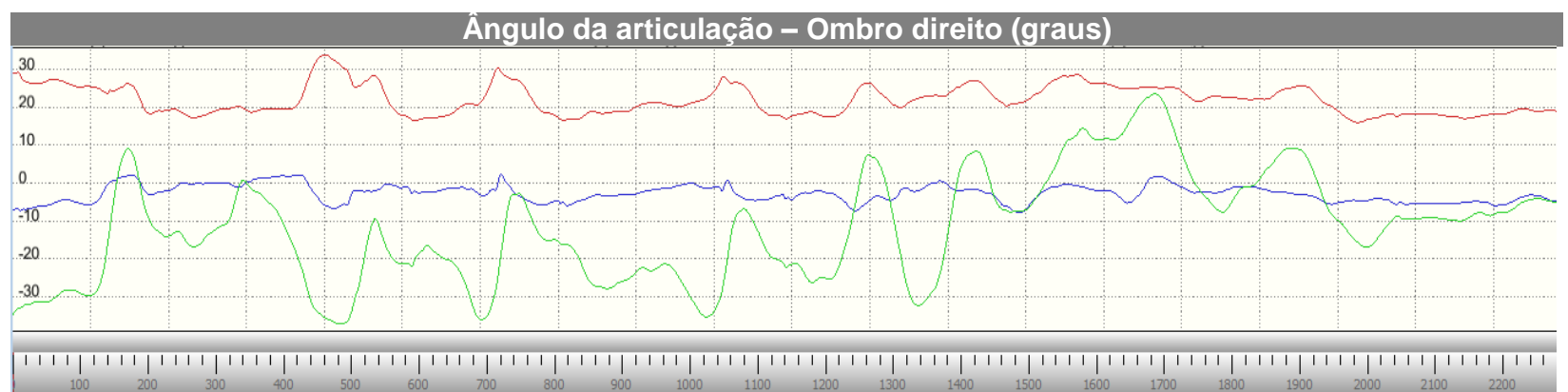

-Abduçăo+)/Aduçăo(-)

-Flexão(+)/Extensăo(-)

- Internal(+)/External(-) Rotação

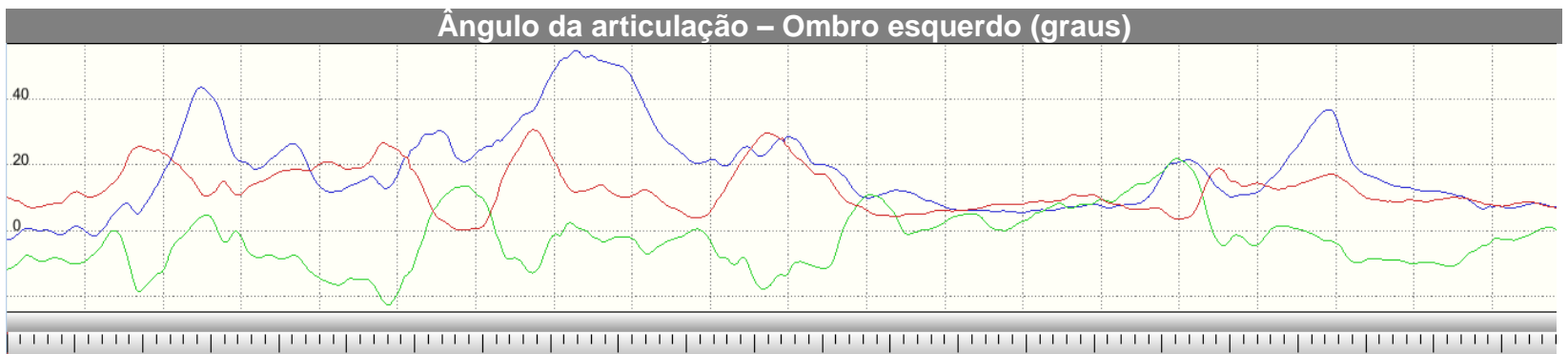

Fonte: os autores.

Com base os dados termográficos das mãos (Figura 7) pode ser observada uma elevação nas temperaturas após a propulsão da cadeira de rodas, sendo $1,1^{\circ} \mathrm{C}$ na mão direita e a $1,7^{\circ} \mathrm{C}$ na mão esquerda. Este aumento pode estar relacionado à pressão aplicada no tecido das mãos durante a propulsão. Segundo Brioschi (2009), uma pressão excessiva aplicada ao tecido que pode gerar mudanças térmicas. 


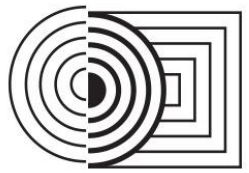

\section{$17^{\circ}$ ERGODESIGN \& USIHC 2019}

PUC-Rio, 11 a 13 de dezembro

Rio de Janeiro, RJ, Brasil $17^{\circ}$ Ergodesign - Congresso Internacional de Ergonomia e Usabilidade de Interfaces Humano Tecnológica: Produto, Informações Ambientes Construídos e Transporte

$17^{\circ}$ USIHC - Congresso Internacional de Ergonomia e Usabilidade de Interfaces Humano Computador

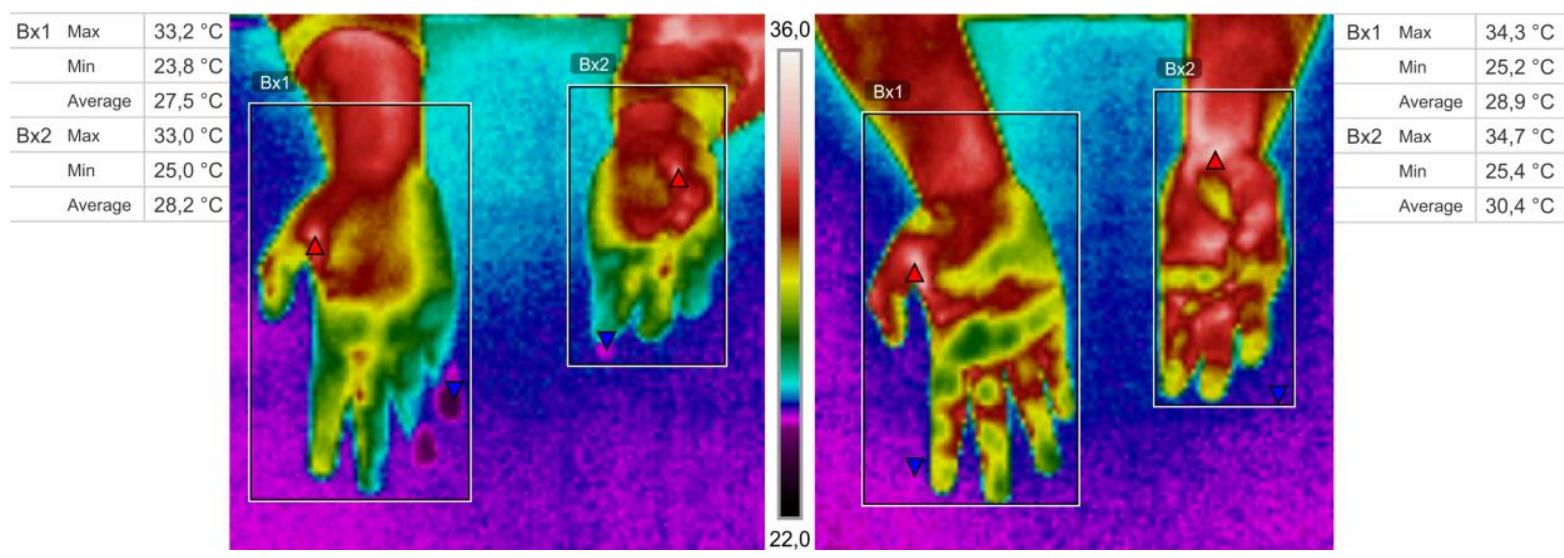

Fonte: os autores.

O ponto hiper radiante da mão direita encontra-se na região tenar, tanto em repouso quanto após a atividade e, na esquerda passou da região tenar (em repouso) para a articulação localizada entre a mão e o punho. Este fato pode estar associado à atrofia existente na mão esquerda, a qual dificulta o manuseio do sistema de propulsão. Diante do exposto, com o objetivo de reduzir a pressão sobre a pele e as articulações, nota-se a oportunidade para o desenvolvimento de um sistema de propulsão diferenciado para a cadeira de rodas, que reduza a necessidade de força, facilitando seu manuseio e diminuindo o atrito com as mãos da usuária.

Mediante a observação dos dados termográficos dos membros inferiores do sujeito (Figura 8) pode-se observar temperaturas mais baixas na região das pernas, o que pode ser associado a menor circulação sanguínea nestes membros que apresentam atrofias musculares. Percebe-se também uma assimetria térmica nos joelhos, destacando a maior temperatura no joelho direito. Além disso, pode ser observada uma redução nas temperaturas após deslocamento no chão, o que pode ser associado ao contato com o piso frio do local. A média da perna direita reduziu $0,9^{\circ} \mathrm{C}$ e a da perna esquerda $1,2^{\circ} \mathrm{C}$.

Figura 8 - Termogramas dos membros inferiores (TR e T0)

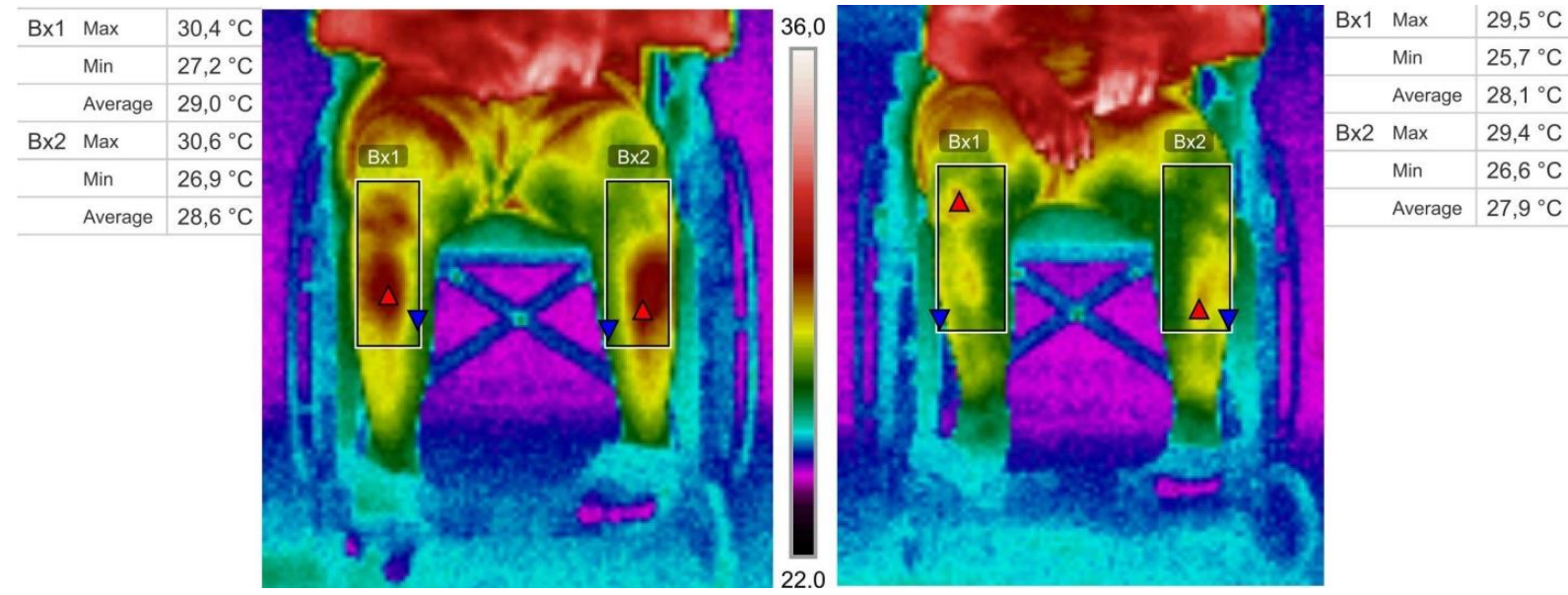

Fonte: os autores. 
Com bases nestas medições é possível identificar as regiões de contato com o piso, que podem orientar o desenvolvimento de um dispositivo para proteção de joelhos e demais regiões que possuem contato com o chão. Essa solução poderia ser combinada com o dispositivo para estabilização e mobilidade, proposto anteriormente, permitindo seu uso combinado ou independente. Esse dispositivo poderia apresentar superfície com materiais confortáveis internamente e resistentes externamente.

Por fim no que tange os dados termográficos da cadeira de rodas (Figura 9), foi observada uma elevação nas temperaturas do assento e encosto após o deslocamento $\left(2,9^{\circ} \mathrm{C}\right.$ no encosto e $4^{\circ} \mathrm{C}$ no assento). Mediante este aquecimento, percebe-se a oportunidade de desenvolver e testar diferentes revestimento para a cadeira de rodas, com o propósito de reduzir o aquecimento e melhorar o conforto térmico da usuária.

Figura 9 - Termogramas da cadeira de rodas (TR e T0).

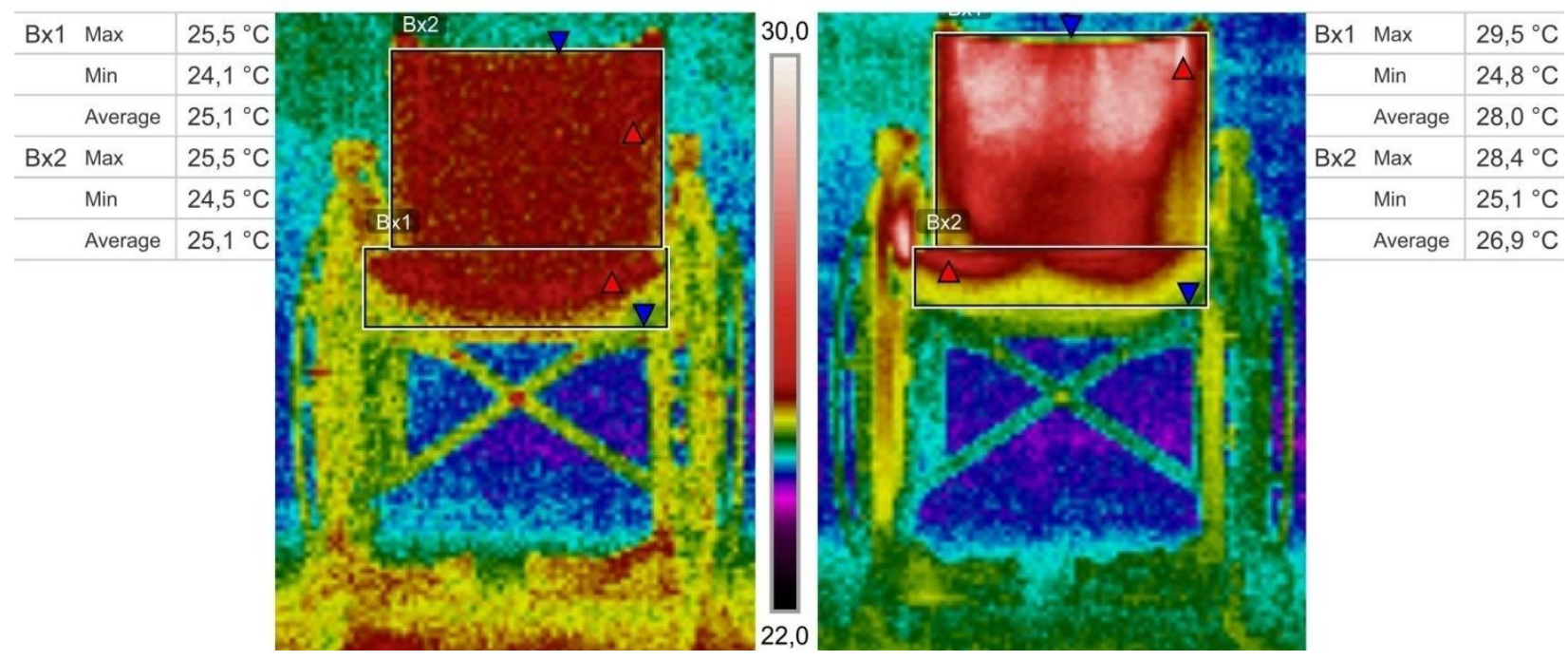

Fonte: os autores.

Além disso, a imagem térmica após a uso do produto demonstrou uma assimetria na temperatura do lado direito e esquerdo do assento, que está associado ao modo como a usuária se posiciona ao sentar na cadeira. Nesse sentido, identifica-se a possibilidade de desenvolver um assento e/ou um encosto personalizado, com base em dados antropométricos da usuária da cadeira de rodas, o qual tornaria a área de contato com o corpo homogênea, aliviando as pressões sobre a pele.

\section{CONCLUSÃO}

Mediante o objetivo de identificar oportunidades para projetos de TAs para um sujeito com deficiência motora a partir da análises de dados termográficos e da captura de movimentos, este estudo demonstrou condições problemáticas relacionadas às posturas do sujeito e ao seu deslocamento, com e sem o uso da cadeira de rodas. 
Com base nas análises realizadas por profissionais da área de design e da saúde, foi possível a identificação de oportunidades de projeto, como: desenvolvimento de um dispositivo assistivo para estabilização e mobilidade, que permita a redução da pressão coxofemorais;

desenvolvimento de um sistema de propulsão diferenciado para a cadeira de rodas, que reduza a necessidade de força, facilitando o manuseio e diminuindo o atrito com as mãos da usuária; desenvolvimento de um dispositivo para proteção de joelhos e demais regiões de contato com o chão durante a marcha; mudança do revestimento da cadeira de rodas, com o propósito de reduzir o aquecimento e melhorar o conforto térmico da usuária; desenvolvimento de um assento e/ou encosto personalizado, com base em dados antropométricos da usuária da cadeira de rodas e; desenvolvimento de um dispositivo que permita a transferência de forma autônoma e segura.

De modo geral, as oportunidades identificadas poderiam auxiliar o sujeito na realização de suas atividades da vida diária, bem como poderiam aumentar seu conforto e segurança, reduzindo 0 risco de lesões e melhorando sua qualidade de vida. No entanto, salienta-se a importância de uma análise mais detalhada do sujeito e de suas necessidades por uma equipe multidisciplinar (fisioterapeutas, médicos, terapeutas ocupacionais, designers, etc.), que possa analisar os problemas por meio de diferentes abordagens e perceptivas.

Em relação aos procedimentos adotados, as observações realizadas contribuíram para visualizar as interações da usuária em diferentes contextos, auxiliando na identificação das suas dificuldades. A termografia infravermelha se mostrou um método eficiente para a coleta de dados com um sujeito com deficiência, destacando-se por seu caráter não invasivo, que permite análises sem a necessidade de contato direto. A captura de movimentos, por sua vez, se mostrou adequada para a simulação real dos movimentos, permitindo definições que contribuíram para o entendimento das necessidades do sujeito. No entanto, cabe ressaltar a importância dos profissionais da saúde para a análise e a interpretação desses dados.

Contudo, como estudos futuros sugere-se a elaboração de pesquisas focadas em cada uma das oportunidades identificadas. Estas pesquisas podem levantar dados específicos sobre cada problema, apresentando requisitos para o desenvolvimento dos projetos.

\section{REFERÊNCIAS BIBLIOGRÁFICAS}

ALVES NETO, O. et al. Dor: princípios e prática. Porto Alegre: Artmed, 2009.

BAUMAN, Zygmunt. Globalização: as conseqüências humanas. Rio de Janeiro: Jorge Zahar, 1999.

BASSO, Liliane. A contribuição do designer no projeto de recursos de tecnologia assistiva: proposta de intervenção colaborativa. 2012. 175 f. Dissertação (Mestrado) - Curso de Design, Universidade Federal do Rio Grande do Sul, Porto Alegre, 2012. Disponível em: $<$ https://www.lume.ufrgs.br/bitstream/handle/10183/62048/000867041.pdf?sequence=1\&isAllow ed=y>. Acesso em: 02 set. 2019.

$\mathrm{BERSCH}, \mathrm{R}$. Introdução à Tecnologia Assistiva. Porto Alegre: Assistiva - Tecnologia e Educação, 2017.

BEST, Kathryn. Fundamentos da gestão do design. Porto Alegre: Bookman, 2012. 
BONNEY, Rosemary A.; CORLETT, Esmond Nigel. Head posture and loading of the cervical spine. Applied ergonomics, v. 33, n. 5, p. 415-417, 2002.

BRASIL. Cartilha do Censo 2010: Pessoas com deficiência. Secretária de Direitos Humanos da Presidência da República (SDH-PR); Secretaria Nacional dos Direitos da Pessoa com Deficiência (SNPD), et al. Brasilia: SDH-PR/SNPD, 2012.

BRASIL. Lei 13.146 de 06 de julho de 2015. Institui a Lei Brasileira de Inclusão da Pessoa com Deficiência (Estatuto da Pessoa com Deficiência). Diário Oficial da União, 2015.

BRIOSCHI, Marcos L.; MACEDO, José F; MACEDO, Rodrigo de A. C. Termometria cutânea: novos conceitos. Jornal Vascular Brasileiro, v. 2, p. 151-60, 2003.

BRIOSCHI et al. Termografia no diagnóstico da dor. In: ALVES NETO, O. et al. Dor: princípios e prática. Porto Alegre: Artmed, 2009.

CERDEIRA, Fernando et al. Applicability of infrared thermography to the study of the behaviour of stone panels as building envelopes. Energy and Buildings, v. 43, n. 8, p. 1845-1851, 2011.

FORCELINI, Franciele; MERINO, Eugenio Andrés Díaz. Termografia Infravermelha no Desenvolvimento de Produtos: formas de abordagem e categorias dos produtos, p. 2665-2678. In: Anais do 13 Congresso Pesquisa e Desenvolvimento em Design (2018). São Paulo: Blucher, 2019. ISSN 2318-6968, DOI 10.5151/ped2018-3.3_ACO_73. Disponível em: $<$ https://www.proceedings.blucher.com.br/article-details/termografia-infravermelha-nodesenvolvimento-de-produtos-formas-de-abordagem-e-categorias-dos-produtos-30139>. Acesso em: 20 ago. 2019.

FORCELINI, Franciele; VARNIER, Thiago; MERINO, Eugenio Andrés Díaz. Termografia Infravermelha e Captura de Movimentos: vantagens e desvantagens no desenvolvimento de projetos, p. 2652-2664. In: Anais do 13 Congresso Pesquisa e Desenvolvimento em Design (2018). São Paulo: Blucher, 2019. ISSN 2318-6968, DOI 10.5151/ped2018-3.3_ACO_72. Disponível em: <https://www.proceedings.blucher.com.br/article-details/termografiainfravermelha-e-captura-de-movimentos-vantagens-e-desvantagens-no-desenvolvimento-deprojetos-30138>. Acesso em: 20 ago. 2019.

FORCELINI, Franciele; MERINO, Giselle Schmidt Alves Díaz. Guia de Orientações Thermos Protocol. Florianópolis: Ngd/Ufsc, 2019. Disponível em: <www.ngd.ufsc.br/thermos-protocol/>. Acesso em: 13 ago. 2019.

FORCELINI, Franciele. Termografia infravermelha aplicada ao design: protocolo de coleta de dados termográficos para o desenvolvimento de projetos. Dissertação (Mestrado em Design) Programa de Pós-Graduação em Design, Universidade Federal de Santa Catarina. Florianópolis, 247 p., 2019.

GOMIDE, João Victor Boechat et al. Captura de movimento e Animação de Personagens em Jogos. In: Brazilian Symposium on Games and Digital Entertainment, 8., 2009, Rio de Janeiro. Simpósio... Rio de Janeiro: PUC, 2009.

GABRIEL, Joaquim et al. Termografia: imagem médica e síndromes dolorosas. Lisboa: Lidel, 2016.

HOLST, Gerald C. Common sense approach to thermal imaging. Washington, DC, USA: SPIE Optical Engineering Press, 2000. 
MARCONI, Marina de Andrade; LAKATOS, Eva Maria. Técnicas de pesquisa: planejamento e execução de pesquisas, amostragens e técnicas de pesquisas, elaboração, análise e interpretação de dados. 6.ed. São Paulo: Atlas, 2007.

MARQUES AP. Ângulos articulares dos membros superiores. In: Manual de Goniometria. 2 ed. São Paulo: Editora Manole. 2003, p.12-17.

MERINO, Eugenio A. D. et al. Implementation of Integrated Instrumentation in Assistive Technology. Advances In Ergonomics In Design, [s.I.], p.549-560, 24 jun. 2017. Springer International Publishing. http://dx.doi.org/10.1007/978-3-319-60582-1_55.

MERINO, Eugenio A. D. et al. O uso da instrumentação tecnológica em projetos de tecnologia assistiva: captura de movimentos e termografia infravermelha. Human Factors In Design, [s.l.], v. 7, n. 14, p.95-113, 15 out. 2018. Universidade do Estado de Santa Catarina. http://dx.doi.org/10.5965/2316796307142018095.

MOBLEY, R. Keith. An introduction to predictive maintenance. 1. ed. Amsterdam: ButterworthHeinemann, 2002.

PALMER, Lynn M.; EPLER, Marcia E. Ombro. In: Fundamentos das Técnicas de Avaliação Musculoesquelética. 2. ed. Rio de Janeiro: Guanabara Koogan; 2000. p.63-108.

SAMPIERI, Roberto H.; COLLADO, Carlos F.; LUCIO, M. P. B. Métodos de Pesquisa. 5. ed. Porto Alegre: Penso, 2013.

SILVA, Júlio Cézar R. P. da; TARALLI, Cibele H.; MELZ, Simone P. M. Termograma: A imagem térmica como instrumento de diagnóstico rápido no design. In: Fourth International Conference on Integration of Design, Engineering and Management for innovation. Anais... Florianópolis, SC, Brasil: 2015. Disponível em: <http://janainaramos.com.br/idemi2015/anais/02/143332.pdf>. Acesso em: 14 set. 2017.

SILVA, Luiz A. da. Termografia: Princípios básicos e suas aplicações. 1. ed. São José dos Campos: Tecnolass Tecnologia, 2017.

SPECK, Giselle Mari et al. Processo de instrumentação integrada no desenvolvimento de projetos de Tecnologia Assistiva. Anais de congresso 18을 Congresso Brasileiro de Ergonomia (ABERGO), Belo Horizonte: 2016.

TILLEY, A. R.; HENRY DREYFUSS ASSOCIATES. As medidas do homem e da mulher : fatores humanos em design. Porto Alegre: Bookman, 2005.

VARNIER, Thiago. Fatores Humanos associados aos projetos de Design: protocolo de coleta para a captura de movimentos. Dissertação (Mestrado em Design) - Programa de Pós-graduação em Design, Universidade Federal de Santa Catarina, Florianópolis, 250 p., 2019.

VARNIER, Thiago; MERINO, Eugenio Andrés Díaz. O Uso da Captura de Movimentos no Desenvolvimento de Produtos: um estudo focado nas tecnologias e aplicações, p. 2463-2477. In: Anais do 13을 Congresso Pesquisa e Desenvolvimento em Design (2018). São Paulo: Blucher, 2019. ISSN 2318-6968, DOI 10.5151/ped2018-3.3_ACO_59. Disponível em: $<$ https://www.proceedings.blucher.com.br/article-details/o-uso-da-captura-de-movimentos-nodesenvolvimento-de-produtos-um-estudo-focado-nas-tecnologias-e-aplicaes-30125>. Acesso em: 20 ago. 2019. 
VARNIER, Thiago; MERINO, Giselle Schmidt Alves Díaz. Motion Capture Protocol: Protocolo de coleta MVN Link Biomech. Florianópolis: NGD/UFSC, 2019. Disponível em: 〈www.ngd.ufsc.br〉. Acesso em: 16 ago. 2019.

WHO. Relatório mundial sobre a deficiência. SEDPcD. São Paulo, p.334. 2012.

XSENS. Moven: user manual. Moven Motion Capture System. The Netherlands: Xsens Technologies B.V. 2012.

YANG, Che-Chang; HSU, Yeh-Liang. A review of accelerometry-based wearable motion detectors for physical activity monitoring. Sensors, v. 10, n. 8, p. 7772-7788, 2010.

\section{AGRADECIMENTOS}

Agradecemos ao sujeito da pesquisa, ao Programa de Pós-graduação em Design da UFSC (PósDesign/UFSC), ao Programa de Pós-Graduação em Engenharia de Produção (PPGEP/UFSC), ao Núcleo de Gestão de Design e Laboratório de Design e Usabilidade (NGDLDU/UFSC), à Rede de Pesquisa e Desenvolvimento em Tecnologia Assistiva (RPDTA), à Universidade Federal da Paraíba (UFPB), à Coordenação de Aperfeiçoamento de Pessoal de Nível Superior (CAPES) e ao Conselho Nacional de Desenvolvimento Científico e Tecnológico (CNPq). 